Anaesthesist 2022 $\cdot 71: 467-474$ https://doi.org/10.1007/s00101-022-01096-w Angenommen: 20. Januar 2022 Online publiziert: 17. Februar 2022 (c) Der/die Autor(en) 2022

\title{
Anästhesiologische Aspekte bei Patienten mit Epidermolysis bullosa
}

\author{
B. Ziegler' $\cdot$ S. Ofner ${ }^{1} \cdot$ J. Lischent ${ }^{1} \cdot$ J. Perndorfer ${ }^{1} \cdot$ A. Diem ${ }^{2,3} \cdot$ M. Laimer ${ }^{2,3}$. \\ C. Prodinger ${ }^{2} \cdot$ F. Treff $^{1}$ \\ ' Univ.Klinik f. Anästhesiologie, perioperative Medizin und allgemeine Intensivmedizin, LKH Salzburg, \\ Salzburg, Österreich \\ ${ }^{2}$ Univ. Klinik f. Dermatologie und Allergologie, LKH Salzburg, Salzburg, Österreich \\ ${ }^{3}$ EB Haus Austria, LKH Salzburg, Salzburg, Österreich
}

\section{Zusammenfassung}

Epidermolysis bullosa führt durch veränderte Strukturproteine der dermoepidermalen Verankerungszone zu Blasenbildung und dadurch zu Vernarbungen und Strikturen an Haut und Schleimhäuten. Patienten mit dieser Erkrankung müssen sich typischerweise häufig chirurgischen Interventionen unterziehen, die durch die Erkrankung und ihre Komplikationen notwendig werden. Die anästhesiologische Betreuung der Betroffenen stellt naturgemäß eine große Herausforderung dar. In diesem Übersichtsartikel werden Besonderheiten dieses Patientenguts und praktische Empfehlungen für seine Betreuung zusammengefasst.

Schlüsselwörter

Verletzlichkeit · Hautblasen · Strikturen · Schwieriger Atemweg · Minimal touch

Unter Epidermolysis bullosa (EB) werden geno - und phänotypisch heterogene Hauterkrankungen, die durch eine moderat bis exzessiv gesteigerte Fragilität der Haut und Schleimhäute gegenüber mechanischen Stimuli gekennzeichnet sind, subsumiert (0 Infobox 1). Die Erstbeschreibungen gehen in das Ende des 19. Jh. zurück. Erste anästhesierelevante Daten sind Ende der 1950-er bzw. Anfang der 1960er-Jahre zu finden. Dabei handelt es sich überwiegend um einzelne Fallberichte [1, 2].

\section{Einteilung, Komplikationen und Begleiterkrankungen}

Mit einer geschätzten Inzidenz von 1:20.000-1:100.000 (Europa und USA) zählt die EB zu den seltenen Erkrankungen („orphan diseases“; D Infobox 2).

Pathophysiologisch liegen der Erkrankung genetisch bedingte Defekte in Strukturproteinen der Dermis und Epidermis zugrunde. Diese beeinträchtigen die Stabilität bzw. die strukturelle und funktionelle
Integrität der Epidermis sowie der dermoepidermalen Junktionszone. Je nach Lokalisation der betroffenen Strukturporteine kommt es zu einer Spaltbildung in variierender Tiefe, anhand derer man 4 EB-Haupttypen unterscheiden kann (• Tab. 1):

- EB simplex (intraepidermale Spaltbildung, 70\% der Fälle),

- junktionale $E B$ (Spaltbildung innerhalb der Lamina lucida, $5 \%$ der Fälle),

- dystrophe EB (dermolytische Spaltbildung) sowie

- Kindler-EB (gemischte Spaltebenen in der Basalmembranzone) [3].

Quantität und Qualität des molekularen Defekts, epigenetische und durch den mutationsbasierten Barrieredefekt ausgelöste sekundär-inflammatorische Prozesse, wie auch Umweltfaktoren haben einen großen Einfluss auf das klinische Bild. Das variable Zusammenspiel dieser Faktoren ist ursächlich für das breit gestreute Symptomenspektrum, welches von vereinzelten Blasen an mechanisch besonders be- 


\begin{tabular}{|c|c|c|c|c|}
\hline EB-Subtyp & $\begin{array}{l}\text { Ebene der } \\
\text { Spaltbildung }\end{array}$ & Vererbung & Mutierte Gene (Proteine) & Klinische Charakteristika \\
\hline \multirow[t]{2}{*}{ EB simplex } & \multirow[t]{2}{*}{ Intraepidermal } & $A D$ & $\begin{array}{l}\text { KRT5, KRT14 } \\
\text { (Keratin 5, 14), PLEC (Plektin), } \\
\text { KLHL24 („Kelch-like member 24“) }\end{array}$ & \multirow{2}{*}{$\begin{array}{l}\text { Häufigste (70\%) und mildeste Form } \\
\text { Oberflächliche Blasen/Erosionen } \\
\text { Verteilung: akral betont, lokalisiert oder auch genera- } \\
\text { lisiert } \\
\text { Verschlechterung durch Hitze, Schwitzen } \\
\text { fallweise Schleimhautläsionen, Muskeldystrophie, } \\
\text { Kardiomyopathie, nephrotisches Syndrom }\end{array}$} \\
\hline & & AR & $\begin{array}{l}\text { KRT5, KRT14 (Keratin 5,14), DST } \\
\text { (,bullous pemphigoid antigen 230“, } \\
\text { BP230), EXPH5 } \\
\text { (Exophilin), PLEC (Plektin), CD151 } \\
\text { (CD151-Antigen) }\end{array}$ & \\
\hline EB junctionalis & $\begin{array}{l}\text { Junktional, } \\
\text { Lamina lucida }\end{array}$ & AR & $\begin{array}{l}\text { LAMA3, LAMB3, LAMC2 (Laminin } \\
\text { 332), COL17A1 } \\
\text { (Typ-XVII-Kollagen), ITGA6, ITGB4 } \\
\text { (Integrin a6ß4), ITGA3 (Integrin, a3- } \\
\text { Untereinheit) }\end{array}$ & $\begin{array}{l}\text { Blasenbildung, chronische Wunden, atrophe Narben- } \\
\text { heilung, Hypergranulation } \\
\text { Nageldystrophie, Alopezie; Zahnschmelzdefekte } \\
\text { Schleimhautkomplikationen (Gastrointestinaltrakt, } \\
\text { Atemwege, Urogenitaltrakt, Augen) }\end{array}$ \\
\hline \multirow[t]{2}{*}{ EB dystrophicans } & \multirow{2}{*}{$\begin{array}{l}\text { Subepidermal } \\
\text { (Sublamina } \\
\text { densa) }\end{array}$} & $A D$ & COL7A1 (Typ-VII-Kollagen) & \multirow{2}{*}{$\begin{array}{l}\text { Chronische Wunden, Milien, Narbenbildung, Fibrose, } \\
\text { Pseudosyndaktylie, ausgeprägter Schleimhautbe- } \\
\text { fall (Gastrointestinal-, Respirations-, Urogenitaltrakt, } \\
\text { Augen), Nageldystrophie, Karies, Wachstumsre- } \\
\text { tardierung, schwere Anämie, Osteopenie/-porose, } \\
\text { Plattenepithelkarzinome }\end{array}$} \\
\hline & & AR & COL7A1 (Typ-VII-Kollagen) & \\
\hline Kindler-EB & Variabel & AR & $\begin{array}{l}\text { FERMT1 (,fermitin family homo- } \\
\log 1^{11)}\end{array}$ & $\begin{array}{l}\text { Akrale Blasenbildung, Fotosensitivität, kutane Atro- } \\
\text { phie, progressive Poikilodermie; Gingivitis; Ösopha- } \\
\text { geale, urogenitale, gastrointestinale Beteiligung }\end{array}$ \\
\hline
\end{tabular}

\section{Infobox 1}

\section{Faktenblatt Epidermolysis bullosa}

- Heterogene Gruppe von Hauterkrankungen

- Inzidenz 1:20.000-1:100.000: „seltene Erkrankung"

- Genetisch bedingte Defekte von Strukturproteinen in Dermis/Epidermis

- Pathologische Fragilität der Haut und Schleimhäute

- Transdermale Protein- und Flüssigkeitsverluste, transkutanes Infektionsrisiko

- Kutane und extrakutane Manifestationen, u.a.

- Chronische Wundareale, erhöhtes Malignomrisiko (Plattenepithel-Ca)

- Zahnschmelzdefekte, Karies, exzessive Vernarbungen und Verwachsungen, Anämie, Kachexie, Immunschwäche, kardiale/renale Organschäden

- Psychische Belastung

- Kausale Therapie (Genmodulation) noch in Erforschung, bisher nur als experimenteller Ansatz

- Prophylaxe/Vermeidung von Traumen jeglicher Art

- Symptomatische Therapie von Wunden, Erosionen, Infektionen und Vernarbungen/Strikturen

\section{Infobox 2}

Seltene Erkrankungen (,orphan diseases"), OrphanAnesthesia

In Europa gilt eine Erkrankung als selten, wenn nicht mehr als 5 von 10.000 Menschen das spezifische Krankheitsbild aufweisen. Rund 30.000 Krankheiten sind weltweit bekannt, davon zählen etwa 8000 zu den seltenen Erkrankungen, auch Orphan diseases genannt. Etwa $80 \%$ der seltenen Krankheiten sind genetisch bedingt, daher machen sich viele schon bei der Geburt oder im frühen Kindesalter bemerkbar. OrphanAnesthesia (www. orphanaesthesia.eu) ist ein Projekt des Wissenschaftlichen Arbeitskreises der DGAI (Deutsche Gesellschaft für Anästhesiologie und Intensivmedizin), welches allen Ärzten, Patienten und Selbsthilfeorganisationen über 200 standardisierte, im Peer-Review-Verfahren überprüfte Handlungsempfehlungen zur anästhesiologischen Betreuung von Patienten mit seltenen Erkrankungen zur Verfügung stellt. Auch für die Epidermolysis bullosa existiert eine OrphanAnesthesiaHandlungsempfehlung [3]. lasteten Hautregionen (Akren), bis hin zu disseminiert-generalisierter spontaner Blasenbildung, chronisch-inflammierten, dysbiotischen Wunden und Multisystembeteiligung (u.a. mit Erosionen, Stenosen, Strikturen in Mundhöhle, Ösophagus, Respirations- und Urogenitaltrakt) reicht. Entsprechend umfangreich sind kutane bzw. extrakutane Komplikationen der "Schmetterlingskinder", wie sie umgangssprachlich auch bezeichnet werden. Hierzu zählen Zahnschmelzdefekte, Karies, exzessive Vernarbungen und Verwachsungen von Fingern und Zehen, Anämie, Kachexie, Immunschwäche sowie Organschäden (Kardiomyopathie, renale Defekte). Letztere können als primäre Manifestation (aufgrund der redundanten Expression von betroffenen Proteinen in epithelialisierten Organen) oder auch sekundär bzw. als therapieassoziierte Komplikationen (beispielsweise Eisenüberlagerung durch Bluttransfusionen bei multifaktorieller Anämie) auftreten. Schwere Formen der Hautbarrieredefizienz sind mit den Komplikationen eines signifikanten Protein- und Flüssigkeitsverlustes sowie einem erhöhten transkutanen Infektionsrisiko assoziiert. Eine weitere meist lebenslimitierte Folge bzw. Komplikation dieser Erkrankung sind die Plattenepithelkarzi- 

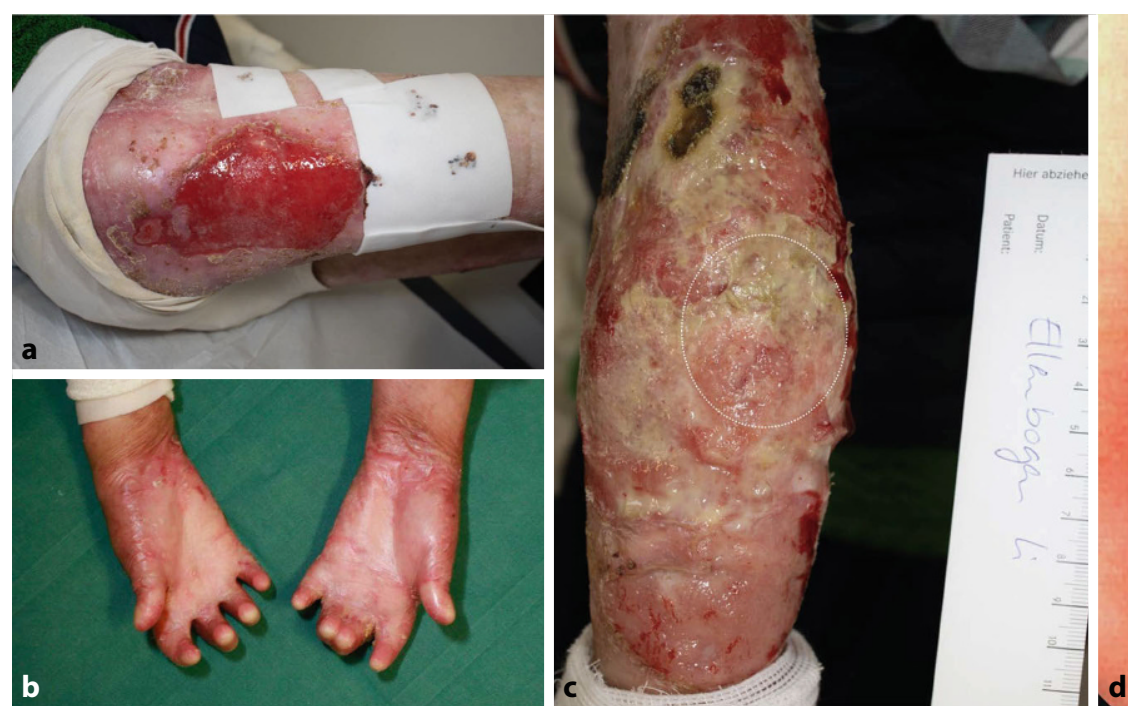

b

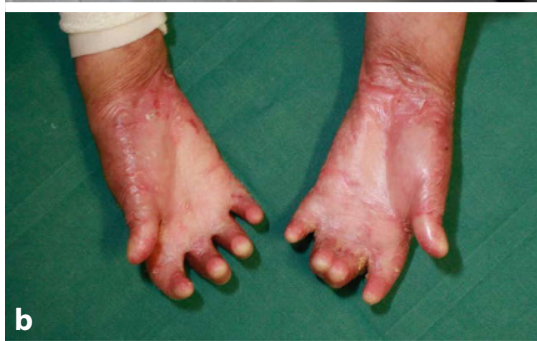

Abb. 1 \ a Verbandwechsel, b Pseudosyndaktylien der Finger, c Plattenepithelkarzinom in chronischer Wunde, d Ösophagusstriktur

nome, die insbesondere in chronischen Wundarealen auftreten [3]. Die Ausprägung dieser Komplikationen trägt zu einer erhöhten Morbidität und Mortalität dieser Patientengruppe bei. Demzufolge ist die Lebenserwartung eingeschränkt und daraus ergibt sich ein Patientengut vorwiegend im Kindes- bis jungen Erwachsenenalter [4].

Die "Schmetterlingskinder" haben neben den physischen Einschränkungen auch eine große psychische Belastung zu tragen. Daher ist es wichtig, den Patienten und deren Umfeld genügend Aufmerksamkeit und Platz zu geben [5].

Eine kausale Therapie ist derzeit trotz vieler hoffnungsvoller Forschungsansätze insbesondere der molekularen Medizin noch nicht möglich. Die Behandlung von EB-Patienten erstreckt sich bislang hauptsächlich auf die Prophylaxe und Vermeidung von Traumen jeglicher Art und die Therapie von Wunden und Erosionen sowie den damit häufig verbundenen Infektionen. Durch Release-Operationen (Lösen von Pseudosyndaktylien) und Bougierungen bzw. Dilatationen (von beispielsweise ösophagealen Engstellen) wird versucht, eine Verbesserung der aus den Vernarbungen entstehenden Komplikationen zu erreichen.

Erfolgversprechend werden aktuell verschiedene Therapieoptionen beforscht [6], zum einen gentherapeutische Strategien, die entweder korrigierte Genabschnit- te transferieren oder gezielt dominant negativ wirkende Mutationen (z. B. über Geneditierung) ausschalten. Weitere Therapieoptionen sind, die lokale oder systemische Substitution von rekombinanten Proteinen und die Produktion von funktionalen Proteinen auf mRNA(messenger ribonucleic acid)-Ebene. Alle diese Therapieoptionen werden derzeit noch in ihrer klinischen Anwendbarkeit evaluiert. Dabei erschweren Effizienzmangel, „Off-target"-Effekte, Gentoxizität (Insertionsmutagenese viraler Vektoren) oder Autoimmunreaktionen auf die "fremden" Neoproteine (bei Patienten mit vorbestehenden Nullmutationen) die Umsetzung translationaler Perspektiven [7].

Merke. Epidermolysis Bullosa kann je nach betroffener Hautschicht in 4 Subgruppen unterteilt werden: EB simplex, junktionale $E B$, dystrophe EB und Kindler-EB. Bisher kann nur symptomatisch durch Vermeidung und Behandlung von Wunden und Infektionen therapiert werden. Die Hoffnung liegt auf der Erforschung von genmodulierenden Therapieansätzen, die bisher jedoch nur experimentell in einigen wenigen Fällen klinisch erfolgreich waren.

\section{Operative Eingriffe}

Die erwähnte Multisystembeteiligung verlangt auch ein spezielles anästhesiologisches Vorgehen bei operativen Eingriffen, das nachfolgend dargestellt wird. In Ermangelung von kontrollierten Studien, beruhen die meisten der nachfolgenden Empfehlungen auf einer Kombination aus evidenzbasierten Erkenntnissen und Expertenkonsens.

Die häufigsten Eingriffe betreffen die Versorgung der kutanen Manifestationen. Hierzu zählen Verbandwechsel, ReleaseOperationen bei Synechien der Finger bzw. Zehen, Hautbiopsien, Resektionen von Hauttumoren (meist Plattenepithelkarzinomen) sowie Ösophagusbougierungen, die aufgrund von Schleimhautstenosen auftreten (• Abb. 1a-d). Auch Zahnsanierungen, die wegen der Zahnschmelzhypoplasie und exzessiver Karies (beachte: schmerzhafte Oralhygiene) in vielen Fällen notwendig sind, können großteils nur unter Allgemeinanästhesie durchgeführt werden. Überdies können alle anderen operativen Eingriffe auch bei EB-Patienten notwendig werden.

Da aus den erwähnten Gründen die Behandlung von EB-Patienten mit einem größeren Aufwand verbunden ist, sollten elektive Behandlungen bzw. Operationen vorwiegend in spezialisierten Zentren durchgeführt werden $[8,9]$.

\section{Anästhesievorbereitung}

Grundsätzlich ist von Beginn an bei einem geplanten operativen Eingriff ein multidisziplinärer Zugang mit Einbeziehung von 


\begin{tabular}{|c|c|c|}
\hline- & Vorrangig bei & Klinik \\
\hline Respirationstrakt & EB junctionalis & $\begin{array}{l}\text { Blasen } \\
\text { Erosionen, Narbenbildung } \\
\text { Schleimhautödeme } \\
\text { Laryngeale Stenosierung, insp. Stridor, Obstruktion } \\
\text { Akute Atemwegsobstruktion (vorrangig in den ersten Lebensjahren) } \\
\text { Destruktion d. Stimmbänder }\end{array}$ \\
\hline $\begin{array}{l}\text { Gastrointestinal- } \\
\text { trakt }\end{array}$ & $\begin{array}{l}\text { EB junctionalis } \\
\text { EB dystrophica }\end{array}$ & $\begin{array}{l}\text { Schleimhauterosionen } \\
\text { Strikturen (ösophageal, anal) } \\
\text { Dysphagie } \\
\text { Pseudodivertikel } \\
\text { Chron. Obstipation } \\
\text { Schmerzhafte Defäkation }\end{array}$ \\
\hline Urogenitaltrakt & $\begin{array}{l}\text { EB junctionalis } \\
\text { EB dystrophica }\end{array}$ & $\begin{array}{l}\text { Dysurie, Hämaturie } \\
\text { Stenosen } \\
\text { Vesikoureteraler Reflux } \\
\text { Hydronephrosen } \\
\text { Urosepsis } \\
\text { Glomerulonephritiden } \\
\text { Renale Hypertension } \\
\text { Amyloidose }\end{array}$ \\
\hline Augen & $\begin{array}{l}\text { EB junctionalis } \\
\text { EB dystrophica }\end{array}$ & $\begin{array}{l}\text { Korneale Erosionen, Narben } \\
\text { Symblepharon } \\
\text { Blepharitis } \\
\text { Ektropiumbildung } \\
\text { Verschluss d, ductus nasolacrimalis }\end{array}$ \\
\hline Mund/Zähne & $\begin{array}{l}\text { EB junctionalis } \\
\text { EB dystrophica }\end{array}$ & $\begin{array}{l}\text { Erosionen an Lippen, Zunge, Mundschleimhaut } \\
\text { Erschwerte Mundhygiene - exzessive Zahnkaries } \\
\text { Vorzeitiger Zahnverlust } \\
\text { Zahnschmelzhypoplasie } \\
\text { Zahndysplasie }\end{array}$ \\
\hline $\begin{array}{l}\text { Hals-Nasen- } \\
\text { Ohren }\end{array}$ & $\begin{array}{l}\text { EB junctionalis } \\
\text { EB dystrophica }\end{array}$ & $\begin{array}{l}\text { Chron. Otitis media/interna } \\
\text { Hörverlust (v. a. bei REBD) }\end{array}$ \\
\hline $\begin{array}{l}\text { Stoffwechsel } \\
\text { Allgemein- } \\
\text { symptome }\end{array}$ & $\begin{array}{l}\text { Bei allen Formen mit } \\
\text { schwerer Ausprägung }\end{array}$ & $\begin{array}{l}\text { Nährstoff-, Flüssigkeits- und Proteinverlust über erosive Hautareale } \\
\text { Beeinträchtigte Nahrungsaufnahme durch gastrointestinale Beteiligung - nutritive Mangelerscheinun- } \\
\text { gen } \\
\text { Katabole Stoffwechsellage } \\
\text { Infektneigung } \\
\text { Chronische Anämie }\end{array}$ \\
\hline
\end{tabular}

Pädiatern, Dermatologen, Chirurgen und Anästhesisten anzustreben. Dabei sollten die Form der EB und die damit verbundenen möglichen Begleiterkrankungen bereits geraume Zeit vor dem geplanten Eingriff erörtert werden [10].

Die für die Anästhesie relevanten systemischen Manifestationen variieren in ihrer Häufigkeit innerhalb der EB-Untergruppen (- Tab. 2). So treten bei der EB simplex generell weniger häufig extrakutane Manifestationen auf als bei anderen Subtypen. Handelt es sich um den junktionalen Subtyp, so ist neben den Schleimhautläsionen des Respirations- und Gastrointestinaltrakts häufig auch mit Anämie, Wachstumsretardierung und Nierenerkrankungen zu rechnen. Bei der dystrophen Form sind das Auftreten von Kardiomyopathien, Glomerulonephritiden, Anämie und die Schleimhautbeteiligung des Gastrointestinal- und Urogenitaltrakts prävalent [6].

Dementsprechend ist bereits bei der anästhesiologischen Visite ein gesamter Status des Patienten zu erheben und dieser bezüglich anästhesierelevanter Begleiterkrankungen einzuschätzen. Die Patienten bzw. deren Angehörige sind üblicherweise bestens mit der Erkrankung und ihrem Gesundheitszustand vertraut, und daher sollten deren Angaben und Anliegen unbedingt ernst genommen und wertgeschätzt werden. Zudem ist es ratsam, nach Möglichkeit die Krankengeschichte v. a. hinsichtlich vorausgegangener Anästhesi- en und deren Protokolle zu sichten und in die Planung miteinzubeziehen [8].

Je nach geplantem Eingriff bzw. dem zu erwartenden Blutverlust und dem Ausgangsblutbild ist bereits bei der Operationsplanung ein entsprechendes Patient Blood Management mit etwaiger vorheriger Optimierung des $\mathrm{Hb}$-Werts zu erwägen [9].

Für dieses besondere Patientengut ist es empfehlenswert, ein spezielles Behandlungsprotokoll zur Anästhesieführung auszuarbeiten, an dem sich alle Mitarbeiter der betreuenden Anästhesieabteilung orientieren können [3, 8]. Dafür hat es sich bewährt, ein eigenes Behandlungsset, das vorbereitete EKG-Elektroden, Pulsoxyme- 


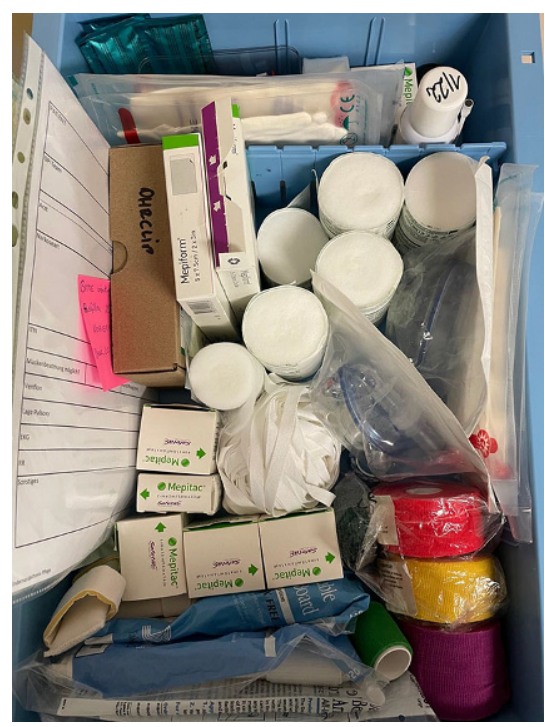

Abb. $2 \Delta$ Anästhesiepflegeset

trie-Clip, Watte, Schaumverbände etc. enthält, zu etablieren (• Abb. 2, [10]).

Einige Eingriffe wie z.B. Bougierungen des Ösophagus, Zahnsanierungen etc. müssen bei den Schmetterlingskindern meist wiederholt durchgeführt werden. Es ist deshalb ratsam, eine Patientenkartei der immer wiederkehrenden Patenten anzulegen. Darin sollten die Prämedikation, der Einleitungsmodus, die Anlagestelle der Venenverweilkanüle, die Atemwegssicherung mit Maßen der Mundöffnung und die Aufrechterhaltung der Narkose dokumentiert werden, um so ein Bild für weitere Narkosen und eine Verlaufsdokumentation der fortschreitenden Erkrankung zu haben.

\section{Prämedikation}

Vielfach sind die Patienten mit Epidermolysis bullosa aufgrund der häufigen medizinischen Eingriffe traumatisiert und profitieren von einer medikamentösen Sedierung vor dem Eingriff [11]. Dafür eignet sich z. B. Midazolam oral in flüssiger Darreichungsform oder - wenn von der Schleimhaut her vertretbar - intranasal mit entsprechendem Applikator. Von einer rektalen Verabreichung ist wegen der verletzlichen Schleimhaut abzusehen.

Merke. Die operativen Eingriffe bei Patienten mit EB betreffen meist die kutanen Manifestationen der Erkrankung, können aber auch alle anderen operativen Eingrif- fe beinhalten. Eine optimale präoperative

Evaluation ist essenziell:

- ausführliche und interdisziplinäre Anamnese mit Berücksichtigung evtl. Begleiterkrankungen,

- Evaluation von Atemweg und Venenzugang,

- Abnahme von Blutproben, z. B. zur Vorbereitung eines Patient Blood Management,

- adäquate Anxiolyse/Prämedikation, da die EB-Patienten meist sehr traumatisiert und ängstlich sind.

\section{Lagerung}

Der Aufrechterhaltung der Haut- und Schleimhautintegrität ist größtes Augenmerk zu schenken, da die Bildung neuer Blasen zu schmerzhaften Wunden, Wärme- und Flüssigkeitsverlust und dem Risiko einer Sekundärinfektion führt [12].

Die Lagerung auf den Op-Tisch muss auf schonende Art und Weise und mit entsprechender Unterpolsterung stattfinden. Es empfiehlt sich, wann immer möglich, Umlagerungen durch aktives Mitwirken des Patienten durchzuführen. Rollboards und ähnliche Umlagerungshilfen, die zu Scherbewegungen führen können, müssen vermieden werden. Generell sollen jegliche Zug - und Scherkräfte im Sinne eines „Notouch" Prinzips vermieden werden. So sind Tischauflage bzw. Stützen und Ablageflächen gut zu unterpolstern und mit Watte einzuwickeln [13-15].

Angesichts des hohen Grades an Kontrakturen sollte der Patient mit den Extremitäten in einer möglichst neutralen Position gelagert werden. Die Wunden sollen geschützt und gepolstert werden, um Blasenbildung oder Drucknekrosen zu vermeiden [10].

Merke. Für eine möglichst schonende Lagerung sollen sich die Patienten im Wachzustand selbst auf dem Op.-Tisch positionieren und alle exponierten Stellen unterpolstert werden.

\section{Monitoring}

Aufgrund der Verletzlichkeit der Haut muss das Anbringen von üblichen Pflastern unbedingt vermieden werden. Ebenso verbietet sich das Anbringen von EKG-Elek- troden oder Pulsoxymetern mit konventionellen Haftstoffen.

\section{Elektrokardiographie}

Es empfiehlt sich, die Klebeflächen großzügig wegzuschneiden und nur den eigentlichen Leiter mit auf Silikonbasis haftenden Schaumverbänden zu fixieren. Alternativ kann auch ein Schlitz in einen besser verträglichen Schaumstoffverband geschnitten werden, wodurch nur der eigentliche Sensoranteil durch die Aussparung Kontakt mit der Haut hat $[9,16]$.

\section{Pulsoxymetrie}

Herkömmliche, selbstklebende $\mathrm{S}_{\mathrm{p}} \mathrm{O}_{2}$-Sensoren, wie sie üblicherweise in der Kinderanästhesie verwendet werden, sind bei diesem Patientengut obsolet. "Clip on"Sensoren für Finger oder Ohren können an entsprechenden Stellen angelegt werden, sofern diese frei von Blasen und Wunden sind. Alternativ können Klebesensoren über Finger geklebt werden, wenn diese Stellen vorher beispielsweise mit einem abgeschnittenen Fingerling eines großen Op.-Handschuhs geschützt werden. Für kürzere Eingriffe wird von manchen Autoren auch die apparative Überwachung nur mittels Pulsoxymetrie toleriert $[4,9]$.

\section{Blutdruckmessung}

Da Druckbelastung normalerweise die Hautintegrität deutlich weniger schädigt als Scherkräfte, kann eine Blutdruckmessung mit durch Watte oder Ähnlichem gut unterpolsterter Blutdruckmanschette durchgeführt werden. Dabei sollte das Messintervall so groß wie möglich gewählt werden und die Indikation für eine invasive Blutdruckmessung zugunsten des Hautschutzes großzügiger gestellt werden.

\section{Temperaturmanagement}

Patienten mit EB sind aufgrund ihrer Hautläsionen und der oft geringen Körpermasse mit einem Mangel an subkutanem Fettgewebe dem Risiko eines gesteigerten Wärmeverlusts ausgesetzt [10]. Deshalb muss für eine entsprechende Raumtemperatur und das aktive Wärmen der 

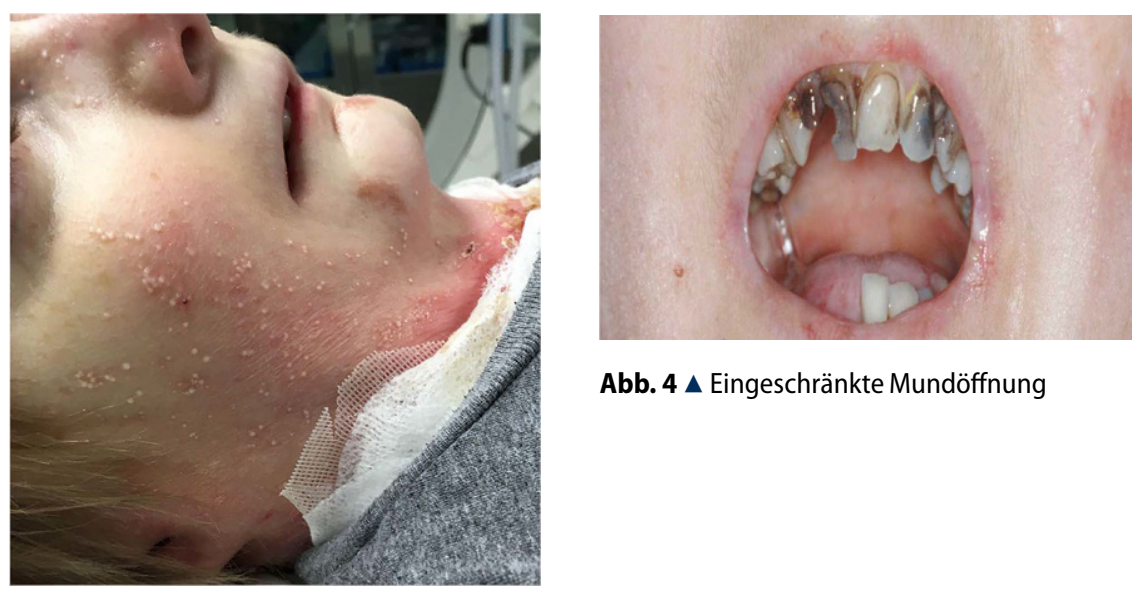

Abb. 4 \ Eingeschränkte Mundöffnung

Abb. $3 \Delta$ Eingeschränkte Beweglichkeit des Unterkiefers

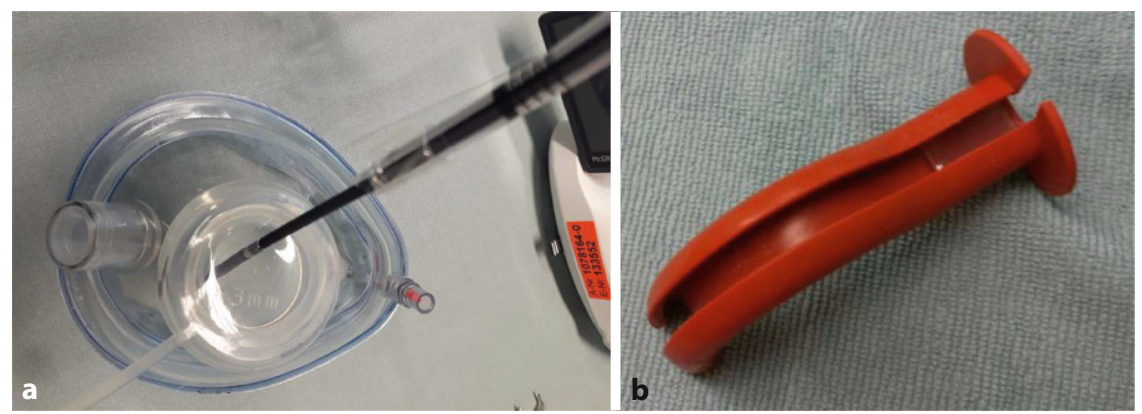

Abb. $5 \Delta$ a Endoskopiemaske, b geschlitzter Guedel-Tubus. Dieser kann nach erfolgreicher Einführung des Intubationsbronchoskopes problemlos entfernt werden

Patienten z.B. durch Wärmematten gesorgt werden. Herkömmliche Temperaturmessungen über nasopharyngeale oder Rektalsonden sind dabei ungeeignet. Am schonendsten gelingt das Temperaturmonitoring über wiederholte Messung mittels Ohrthermometer $[9,12]$.

Merke. Beim Monitoring gilt es, Hautkontakt und v. a. Scher- und Zugkräfte so gut wie möglich zu vermeiden. Das heißt, Monitoring auf ein Mindestmaß reduzieren. Keine selbstklebenden Elektroden oder festhaftenden Pflaster verwenden. Exponierte Areale großzügig mit Watte oder Schaumverbänden fixieren und unterpolstern.

\section{Intravenöser Zugang}

Aufgrund der meist großfächigen Blasenbildung, weitläufigen Vernarbungen und gelenkübergreifender Kontrakturen gestaltet sich das Legen eines i.v.-Zugangs schwer und wird von den Patienten vor dem Einleiten der Narkose oft nicht
Falls die Etablierung eines Venenzugangs vor der Einleitung der Narkose nicht möglich ist oder nicht toleriert wird, bietet sich auch eine inhalative Einleitung an [3]. Dabei ist auf eine prophylaktische Abdeckung der Gesichtspartien mit z.B. Watte oder mit nichtadhäsiven Schaumverbänden zu achten, um etwaige Blasenbildung durch Manipulation des Anästhesisten zu vermeiden $[3,10]$. Probleme bei der Maskenbeatmung können sich dabei evtl. durch eine eingeschränkte Bewegung des Unterkiefers ergeben (- Abb. 3). Generell stellt jedoch die Maskenbeatmung in der Regel kein Problem dar (siehe Abschn. Atemweg).

Speziell EB-Patienten mit Ösophagusstrikturen haben ein erhöhtes Aspirationsrisiko. Deshalb sollte in diesen Fällen eine prophylaktische Gabe von Protonenpumpeninhibitoren in Erwägung gezogen werden $[9,10]$.

\section{Atemweg}

Ein schwieriger Atemweg kann sich aus den anatomischen Veränderungen ergeben: eingeschränkte Mundöffnung durch narbige Strikturen (Mikrostomie, intraorale Strikturen), eingeschränkte Beweglichkeit des Unterkiefers und des gesamten Nackens, Überbiss (Prognathie) im Bereich des Kiefers sowie Verletzlichkeit der Haut im Gesicht und der Schleimhäute des Respirationstraktes (• Abb. 4) . Während diese Hindernisse für das Atemwegsmanagement in den ersten Lebensjahren noch weniger ausgeprägt sind, kann es mit zunehmendem Alter und progredienter Vernarbung zu einer Aggravierung dieser Faktoren kommen und sich damit das Atemwegsmanagement zunehmend herausfordernd gestalten. Demgegenüber kann es bei sehr ausgeprägten Formen der junktionalen EB bereits in den ersten Lebensjahren zu einer tracheotomiepflichtigen Atemwegsobstruktion kommen.

Eine eingeschränkte Beweglichkeit der Zunge (Ankyloglossie) kann einerseits die Laryngoskopie/Intubation erschweren, erleichtert andererseits jedoch die Maskenbeatmung [9, 10, 13]. Eine Engstelle im Bereich der Glottis durch vorausgegangene Atemwegsmanipulationen kann zu weiteren Schwierigkeiten bei der Atemwegssicherung führen. Eine präoperative 
Evaluierung mittels Mallampati-Score ist bei den oft kleinen Patienten nicht immer eindeutig und daher nur bedingt aussagekräftig.

Beim EB-Patientengut, das in den letzten 18 Jahren an der Universitätsklinik für Anästhesiologie perioperative Medizin und allgemeine Intensivmedizin des Landeskrankenhaus Salzburg anästhesiert wurde, hat sich eine durchschnittliche Mundöffnung (gemessen nach Narkoseeinleitung und Relaxierung) von $11 \mathrm{~mm}$ ergeben. Seit dem flächendeckenden Einsatz der Videolaryngoskopie hat sich die primäre Erfolgsrate der trachealen Intubation auch bei dieser Patientengruppe deutlich verbessert. Dabei sollen Spatel und sonstige Instrumente/Gerätschaften, die mit der (Schleim-)Haut in Berührung kommen, mit Gleitgel vorbehandelt werden. Die Tubusgröße (ID) sollte ca. eine halbe Nummer kleiner gewählt werden, als es dem jeweiligen Alter entspricht. Alternativ kann eine endoskopische Intubation nasal oder oral mithilfe einer Endoskopiemaske und eines geschlitzten Guedel-Tubus erfolgen (• Abb. 5). Dies wird jedoch nach unserer Erfahrung nur nach bereits erfolgter Einleitung toleriert und bedarf neben eines Anästhesisten noch mindestens 2 erfahrener Pflegekräfte. Die Platzierung eines Trachealtubus führt dabei kaum zu Schädigungen der Trachealschleimhaut, wohingegen es im oropharyngealen Bereich, v. a. bei unvorsichtigen Manipulationen, sehr wohl zur Ausbildung neuer Blasen kommen kann [17].

Eine weitere Möglichkeit besteht im initialen Einsatz einer Larynxmaske [12] (eine Nummer kleiner als üblich). Über diese kann bei Bedarf auch eine fiberoptische Intubation erfolgen. Von einer länger andauernden Maskenbeatmung wird aufgrund der meist unvermeidlichen Druckschädigungen abgeraten.

Die Tubusfixation erfolgt am besten mit Verbandgaze, die ebenfalls mit Watte o. Ä. unterpolstert wird.

Zum Augenschutz sollten die Augen verschlossen und mit einem Schaumverband fixiert werden. Augensalben werden von den meisten Patienten nicht gut toleriert, was zu verstärktem Auswischen der Augen nach dem Aufwachen und damit er- höhtem Risiko einer Hornhautschädigung führt [16].

Merke. Fortschreitende Vernarbung von Nasopharynx und Larynx erschwert mit steigendem Lebensalter zunehmend den Zugang zu den Atemwegen. Durch Einsatz der Videolaryngoskopie hat sich die Rate der primär erfolgreichen Intubationen bei diesen Patienten deutlich verbessert. Um Verletzungen zu vermeiden, sollten Spatel mit Gleitgel benetzt und die Tubusgröße eine Nummer kleiner gewählt werden. Alternativ ist die fiberoptische Intubation mit einem geschlitzten GuedelTubus oder über eine Larynxmaske (eine Nummer kleiner wählen) möglich.

\section{Aufrechterhaltung der Narkose}

Die Aufrechterhaltung der Narkose als balancierte oder i.v.-Anästhesie kann analog zu üblichen Narkosen durchgeführt werden. EB-Patienten leiden oft an postoperativer Übelkeit und sollten deshalb bereits intraoperativ eine antiemetische Prophylaxe erhalten.

\section{Regionalanästhesie}

Regionalanästhesiologische Verfahren (sowohl rückenmarknahe als auch periphere Blockaden) stellen als begleitende Maßnahme oder bei entsprechend kooperativen Patienten auch als alleiniges Anästhesieverfahren eine sehr gute Möglichkeit dar. Dabei sollte auf lokale Infiltrationen verzichtet werden, um die Entwicklung weiterer Blasen durch die Quaddelbildung zu verhindern [3, 14, 18]. Ebenso sollte die Desinfektion ohne Wischbewegungen durchgeführt werden und eher mit Aufsprühen oder Tupfen des Desinfektionsmittels gearbeitet werden.

\section{Ausleitung der Narkose/ Extubation}

Grundsätzlich ist eine tief schlafende Extubation möglich. Voraussetzung dafür sind allerdings eine komplikationslose Maskenbeatmung und leichte Intubation. Ist dies nicht gegeben, sollten die Patienten wach extubiert werden. Dies wiederum birgt das Risiko, dass durch etwaiges Pressen und Würgen während der Extubation Schleim- hautläsionen entstehen können. Eine enorale Absaugung ist restriktiv und vorsichtig durchzuführen. Dabei sollte ein direkter Schleimhautkontakt tunlichst vermieden werden. Patienten mit Eingriffen am Ösophagus (Dilatation etc.) oder im Bereich der Atemwege sollten intraoperativ Dexamethason, 0,25-0,5 mg/kgKG (max. 8-12 mg), i.v. als abschwellende Maßnahme erhalten $[10,13]$.

\section{Postoperative Phase}

Nach Beendigung des Eingriffs und Extubation sollte wieder eine achtsame Umlagerung des Patienten erfolgen. Konventionelle Sauerstoffmasken sollten wegen der scharfkantigen Oberfläche nach Möglichkeit vermieden und eine adäquate $0 x y$ genierung bei Bedarf durch das Vorhalten einer gepolsterten Beatmungsmaske erreicht werden.

Analgetisch hat sich ein multimodales Konzept bewährt, dass sowohl NSAR als auch Opioide beinhaltet. Allerdings sollten juckreizbegünstigende Opioide wie z. B. Morphin vermieden werden [19]. Als Verabreichungsform wird bei Schluckbeschwerden eine flüssig-orale oder i.v.Form bevorzugt. Eine rektale Gabe ist aufgrund des Risikos einer Schleimhautläsion obsolet [9].

Eine gute Form der Verabreichung von Opioiden ist die „patient controlled analgesia" (PCA). Diese kann entweder vom Patienten selbst oder von einem Angehörigen oder einer Pflegefachkraft bedient werden [19].

Neben der i.v.-Form können Opioide auch in einigen Fällen intranasal oder bukkal verabreicht werden. Allerdings setzt dies eine intakte Schleimhaut an diesen Lokalisationen voraus [19]. Außerdem sind Regionalanästhesieverfahren eine gute Kombinationsmöglichkeit zu i.v.- oder p.o.-Analgesie.

Merke. Die Betreuung von Patienten mit Epidermolysis bullosa ist komplex und bedarf eines interdisziplinären Vorgehens unter Miteinbeziehung von Pädiatern, Dermatologen, Chirurgen und Anästhesisten. Die Anästhesie bei diesen Patienten sollte in spezialisierten Zentren durchgeführt werden. Ein Eingriff sollte gut geplant und die präoperative Evaluierung unter Berück- 
sichtigung aller Begleiterkrankungen und Komplikationen der EB durchgeführt werden. Für Einrichtungen, die regelmäßig diese Patienten behandeln, stellt die Führung einer Patientenkartei mit maßgeblichen Informationen über bereits stattgehabte Eingriffe sowie Behandlungskits mit allen benötigten Materialen eine wertvolle Hilfe dar. Bei allen Interventionen und Therapien an diesen Patienten sind jegliche Traumata, Friktionen und Druckstellen an Haut und Schleimhaut durch Polsterung und adäquate Lagerung bestmöglich zu vermeiden. Postoperativ sollte auf eine suffiziente multimodale Schmerztherapie geachtet werden.

\section{Korrespondenzadresse}

\section{B. Ziegler}

Univ.Klinik f. Anästhesiologie, perioperative Medizin und allgemeine Intensivmedizin, LKH Salzburg

Müllner Hauptstr. 48, 5020 Salzburg, Österreich b.ziegler@salk.at

Funding. Open access funding provided by Paracelsus Medical University.

\section{Einhaltung ethischer Richtlinien}

Interessenkonflikt. B. Ziegler, S. Ofner, J. Lischent, J. Perndorfer, A. Diem, M. Laimer, C. Prodinger und F. Treff geben an, dass kein Interessenkonflikt besteht.

Für diesen Beitrag wurden von den Autoren keine Studien an Menschen oder Tieren durchgeführt. Für die aufgeführten Studien gelten die jeweils dort angegebenen ethischen Richtlinien.

Open Access. Dieser Artikel wird unter der Creative Commons Namensnennung 4.0 International Lizenz veröffentlicht, welche die Nutzung, Vervielfältigung, Bearbeitung, Verbreitung und Wiedergabe in jeglichem Medium und Format erlaubt, sofern Sie den/die ursprünglichen Autor(en) und die Quelle ordnungsgemäß nennen, einen Link zur Creative Commons Lizenz beifügen und angeben, ob Änderungen vorgenommen wurden.

Die in diesem Artikel enthaltenen Bilder und sonstiges Drittmaterial unterliegen ebenfalls der genannten Creative Commons Lizenz, sofern sich aus der Abbildungslegende nichts anderes ergibt. Sofern das betreffende Material nicht unter der genannten Creative Commons Lizenz steht und die betreffende Handlung nicht nach gesetzlichen Vorschriften erlaubt ist, ist für die oben aufgeführten Weiterverwendungen des Materials die Einwilligung des jeweiligen Rechteinhabers einzuholen.

Weitere Details zur Lizenz entnehmen Sie bitte der Lizenzinformation auf http://creativecommons.org/ licenses/by/4.0/deed.de.

\section{Anesthesiological aspects in patients with epidermolysis bullosa}

Epidermolysis bullosa causes blistering due to altered structural proteins of the dermoepidermal junction, resulting in scarring and strictures of the skin and mucous membranes. Affected individuals typically require frequent surgical interventions due to burdensome symptoms and complications of the disease. The anesthesiological management of these patients is inherently challenging. This review article summarizes the relevant features of this patient cohort and provides practical recommendations for care.

\section{Keywords}

Vulnerability $\cdot$ Blister $\cdot$ Stricture $\cdot$ Difficult airway $\cdot$ Minimal touch

\section{Literatur}

1. Koaota Y, Norton ML, Goldenberg S (1961) Anesthetic management of patients with epidermolysis bullosa undergoing surgery. Anesth Analg 40(2):244-250

2. Wilson $F$ (1959) Epidermolysis bullosa: a rare disease of anaesthetic interest. Br J Anaesth 31:26-31

3. Kaltofen H, Steinmann D, Bergmann J (2020) Anaesthesia recommendations for Epidermolysis bullosa. Orphananeshtesia.eu

4. Griffin (1993) The anaesthetic management of patients with dystrophic epidermolysis bullosa. Anesthesia 48:810-815

5. Has C, Liu L, Bolling MC, Charlesworth AV, Hachem ME, Escamez MJ et al (2020) Clinical practice guidelines for laboratory diagnosis of epidermolysis bullosa. Brit J Dermatol 182:574-592

6. Laimer M, Lanschützer CM, Nischler E, Klausegger A, Diem A, Pohla-Gubo G, Bauer JW, Hintner H (2009) Erbliche Blasenbildende Erkankungen, Klinik, Diagnosik und Therapie der Epidermolysis Bullosa. Hautarzt 60:378-388

7. Welponer T, Prodinger C, Pinon-Hofbauer J, Hintersteininger A, Breitenbach-Koller H, Bauer J, Laimer M (2021) Clinical Perspectives of GeneTargeted Therapiesfor Epidermolysis Bullosa. Dermatol Ther 11:1175-1197

8. Herod J, Denyer J (2002) Epidermolysis Bullosa in children. Pathophysiology, anaesthesia and pain management. Paediatr Anaesth 12:388-397

9. Bowen L, Burtonwood MT (2018) Anesthetic management of children with epdermolysis bullosa. BJAEduc 18(2):41-45

10. Goldschneider K, Lucky AW, Mellerio JE, Palisson F, Miranda MC, Azizkhan RG (2010) Perioperative care of patients with epidermolysis bollosa: proceeings of the 5 th international symposium on epidermolysis bullosa, Santiago Chile, December 4-6, 2008. Pediatr Anesth 20:797-804

11. Gottschalk A, Venherm S (2010) Anesthesia for ballon dilatation of esophageal strictures in children with epidermolysis bullosa dystrophica. Curr Opin Anaesthesiol 23(4):518-522

12. Nandi R, Howard R (2010) Anesthesia and epidermolysis bullosa. Dermatol Clin 28:319-324

13. Saraf VS, Mandawade NJ, Gore SK, Padhye UD, Pereira CS (2013) Epidermolysis bullosa: careful monitoring and no touch principle for anesthesia management. J Anaesthesiol Clin Pharmacol 29(3):390-393

14. Ames WA, Mayou BJ (1999) Anaesthetic management of epidermolysis bullosa. Br J Anaesth 82(5):746-751
15. lohom G, Lyons B (2001) Anaesthesia for children with epidermolysis bullosa: a review of 20 years' experience. Eur J Anaesthesiol 18:745-754

16. Knab J, Schumann H (2010) Anästhesiol Intensivmed Notfallmed Schmerzther 45:618-624

17. Holzman RS, Worthen HM (1987) Anaesthesia for children with junctional epidermolysis bullosa. Can JAnaesth 34:395-399

18. van den Heuvel I, Gottschalk A (2016) Feasibility, efficacy, and safety of ultrasound-guided axillary plexus blockade in pediatric patients with epidermolysis bullosa dystrophica. Ped Anesth 26:405-408

19. Goldschneider K, Good J, Harrop E (2014) Pain care for patients with epidermolysis bullosa: best care practice guidelines. BMCMed 12:178 